\title{
Intravenous Antibiotic: Potential Alternative for Restricted Oral Route during Removal of Third Molar
}

\author{
Chidambaram Ramasamy ${ }^{1}$ and Dhanavel Jawahar ${ }^{2}$ \\ 'Department of Prosthodontics, Lecturer, Faculty of Dentistry, AIMST University, Jalan Bedong-Semeling, 08100, Bedong, Kedah Darul Aman-Malaysia. \\ ${ }^{2}$ Reception \& Primary Care Unit, Faculty of Dentistry, AIMST University, Jalan Bedong-Semeling, 08100, Bedong, Kedah Darul Aman-Malaysia. \\ Correspondence : \\ Dr. Chidambaram Ramasamy \\ Department of Prosthodontics, Lecturer, Faculty of Dentistry, AIMST University, Jalan Bedong-Semeling, 08100, Bedong, Kedah Darul Aman-Malaysia. \\ E-mail: dr.ramasamyc@gmail.com \\ DOI: 10.5530/jyp.2016.1.14
}

\section{Dear Sir,}

Third molar popularly called as wisdom tooth has been a prime concern for oral and maxillofacial surgeons owing to its associated problems. The failure to erupt in oral cavity, because of mechanical obstruction leads to impaction (fusion) of tooth with jaw bone. Conservative care of the impacted tooth is not feasible, considering its unfavourable anatomy. Thus surgical removal of tooth remains the gold standard for impacted teeth. Furthermore, a host of factors such as occurrence of pericoronitis (localised infection surrounding wisdom tooth), secondary caries and debris lodgement complicate the scenario thereby requiring prompt service. Irrespective of the strict protocols undertaken, post-operative complications (alveolar osteitis and wound infection) have been reported in candidates. ${ }^{1}$ In order to control the wound infection, oral clinicians have been pushed to administer antibiotic via oral or intravenous route. Literature contains ample evidence on the former mode of delivery with an unclear summary. ${ }^{1}$ Meanwhile, neither a controversy prevails nor do valid documents exist on intravenous antibiotics. Less has been discussed on the impact of IV antibiotics in dental tributaries. This could be the possible reason for being unpopular among oral physicians. Current letter addresses the indications of IV antibiotics during third molar surgery to restore its unsupported preference.

In emergency circumstances (critical illness), oral delivery is restricted and demand prompt service for patients with severe infections. The potential alternative for oral route in such crisis is IV antibiotics. Indications of IV antibiotics are more applicable to immune compromised patients considering their increased risk to bacteremia. Oral intake is mutually affected because of ill- health and preference of IV route serves the purpose. At the same time, antibiotic prescription should be at reserve for high-risk candidates which include indwelling central venous catheter, prosthetic joint and compromised health. ${ }^{2-5}$ Earlier researches have preferred Penicillin (1g IV/ 1 hr pre-op), Clindamycin (300 mg IV/ $1 \mathrm{hr}$ pre-op) and Metronidazole (1g IV/ $1 \mathrm{hr}$ pre-op) in the clinical trials. ${ }^{1}$ Former historical drug is popular among health care professionals because of improved performance over the Streptococci and long half- life. Candidates allergic to Penicillin could benefit from Clindamycin which is equally effective against anaerobes and Streptococci. The concentrations of Clindamycin reach peak level in tissue, thus enabling it to be indicated before minor oral surgical procedures. ${ }^{6}$ Metronidazole's impelling resistance against the anaerobes responsible for majority of odontogenic infection enrol it among the standard IV drugs.

Unique feature of IV antibiotic being quick absorption helps to attain peak level in surgical site immediately after the onset of injection. Hence forth, maintaining its potency throughout the surgical manoeuvre. A vital concern during their delivery is dosage timing which indirectly influences the treatment prognosis. Delayed administrations hinder the drug potential and fail to reduce the post-surgical infection. Bottom line of antibiotic prophylaxis is to choose the right drug and maintain drug level in tissue priorlafter surgical procedure. IV antibiotic satisfy the stated principles and minimise post-operative infections when administered two hours prior the procedure..$^{7}$ Additional elements which influence in choosing IV administration are its associated risk for bacteremia and involved expenses. Even though post-operative bacteremia sustains less than quarter hour after bleeding, clinical prognosis relies on degree of trauma, duration consumed to recover with the least evidence of infections at surgical site.

On a general note, periodic maintenance of drug-kit is essential to avert the usage of expired medication and contaminated needles. Later error could transport pathogens of infectious diseases like Hepatitis B, C and Human Immunodeficiency Virus (HIV). The well utilised short-term benefits of IV antibiotics could metamorphose to long-term consequences on abuse. Inessential prescription of antibiotics may endanger the prevailing medical status and rather induce anti-microbial resistance. ${ }^{8-10}$ In the interest of patient's well-being, performing the dentoalveolar surgery in medical hospital would be still secure. Though dental literature has not fed us with recent inputs on IV antibiotic for more than a decade, the rapid bio-availability in bloodstream reminds its superior efficacy over the oral route. It's on good belief that our communication would motivate the oral physicians to reconsider IV antibiotics and undertake new tasks in near future

\section{REFERENCES}

1. Willian Caetano Rodrigues, Roberta Okamoto, Eduardo Piza Pellizzer, Ana Cláudia Nazareno dos Anjos Carrijo, Rafael Santiago de Almeida, et al. Antibiotic prophylaxis for third molar extraction in healthy patients: current scientific evidence. Quintessence Int. 2015; 46(2): 149-61.

2. Ramasamy C. A cautionary tale on the central venous catheter: medical manual for oral physicians. Malay. J. Med. Sci. 2015; 22(5): 76-84.

3. Ramasamy C. Optimal antibiotic dosage for chronic kidney disease patient in dental office: pharmacological manual for oral clinicians. Recent Pat. Antiinfect. Drug Discov. 2015: 10(2): 113-23.

4. Ramasamy C. Dentist-Nephrologist symbiosis in the dental management of chronic kidney disease patient. J. Coll. Physicians Surg. Pak. 2015; 24(12): 955.

5. Ramasamy C. Dentist-Urologist interplay in the dental management of penile prosthesis patient. J. Coll. Physicians Surg. Pak. 2015; 25(8): 628.
6. Mueller SC,Henkel KO, Neumann J, Hehl EM, Gundlach KK, Drewelow B. Perioperative antibiotic prophylaxis in maxillofacial surgery: penetration of clindamycin into various tissues. J Craniomaxillofac Surg. 1999; 27(3): 172-6.

7. Capuzzi P, Montebugnoli L, Vaccaro MA. Extraction of impacted third molars: a longitudinal prospective study. Oral Surg. 1994; 77(4): 341-3.

8. Ramu C, Padmanabhan TV. Indications of antibiotic prophylaxis in dental practice. Asian Pac J Trop Biomed. 2012; 2(9): 749-54.

9. Ramasamy C. Final thoughts on antibiotic use: wake up call for the oral health care professionals. Asian Pac J Trop Biomed. 2014; 4(Suppl 2): 554-59.

10. Ramasamy C. Cardinal decision for an endodontist to make: is not only, in which infection to use antibiotic, but whether to use one at all.? Asian J Pharm Clin Res. 2014; 7(Suppl 1): 4-5. 